\title{
Full-Mouth Implant-supported Rehabilitation with a Flapless Surgical Technique: A Treatment Approach using Computer-Assisted Oral Implant Surgery
}

\author{
${ }^{1}$ Eitan Mijiritsky, ${ }^{2}$ Adi Lorean, ${ }^{3}$ Horia Barbu, ${ }^{4}$ Ziv Mazor \\ ${ }^{1}$ Department of Oral Rehabilitation, The Maurice and Gabriela Goldschleger School of Dental Medicine, Tel Aviv University, Israel \\ ${ }^{2}$ Private Practice, Haifa, Israel \\ ${ }^{3}$ Lecturer, Faculty of Dental Medicine, Department of Oral Implantology, Titu Maiorescu University, Bucharest, Romania \\ ${ }^{4}$ Private Practice, Raanana, Israel
}

Correspondence: Eitan Mijiritsky, Moshe-Sne Str 22A, Tel Aviv-69350, Israel, Phone: 972-3-6449139, Fax: 972-3-6449137 e-mail: mijiritsky@bezeqint.net

\section{ABSTRACT}

Successful implant treatment includes osseointegration as well as prosthetically optimal positions of the implants for esthetics and function. Computer-assisted oral implant surgery offers several advantages over the traditional approach. The purpose of this report was to evaluate a complex case of a 28-year-old female patient who lost all her teeth as a result of a aggressive periodontal disease resulting in severe vertical and horizontal bone loss. The patient underwent a bilateral open sinus lift procedure and after 6 months dental implants were placed in both jaws with the help of computerized tomography (CT)-based software planning and computer-assisted manufacture of a laboratory-based acrylic surgical guide. A total of 13 dental implants were placed in both jaws using a flapless approach followed by immediate loading of the implants and implant-supported full arch fixed dentures.

The CT-based software program and surgical stents contributed to the success of this case.

Keywords: Computer-assisted surgery, Dental implants, Flapless implant surgery, Immediate loading, Stereolithography, Surgical template.

\section{INTRODUCTION}

Surgical placement of dental implants is a well documented treatment for edentulism. Treatment success rates are high and postoperative complications relatively modest. ${ }^{1-3}$ Further enhancements in treatment modalities have included immediate loading and placement without flap elevation to increase patient comfort and acceptance. The advantages of flapless procedures include reduced intraoperative bleeding and decreased postoperative patient discomfort. ${ }^{4-5}$ However, flapless implant surgery has generally been perceived as a blind procedure because of the difficulty in evaluating alveolar bone shape and angulation. ${ }^{6}$ The loss of bone width cannot be determined on a two-dimensional traditional radiograph and can be difficult to evaluate clinically. Successful implant treatment involves osseointegration of implants that are placed in ideal positions for fabrication of a dental prosthesis. Preoperative prosthetic planning is crucial for a successful treatment outcome. ${ }^{7}$ Computerized tomography (CT) scanning can provide important information and can be used to more accurately plan implant placement. Also, software can render CT data and implant simulation can be performed, facilitating treatment planning as well as implant selection. To transfer a simulated implant position to the surgical field, guidance methods have been developed. ${ }^{8}$ Surgical guidance utilizes CAD/CAM technology to generate guides that incorporate drilling housings for precise placement of implants according to preoperative plans. A few systems are available that allow fabrication of a computer-generated surgical guide. ${ }^{9}$ During the planning phase, a CT scan is obtained with a radiographic template in place; the practitioner performs implant-planning with dedicated software. Once implant selection and positioning have been determined, the radiographic guide serves as a surgical guide with incorporated drill housing, by the aid of a positioning device. ${ }^{10}$ This report illustrates the flapless treatment of a complex case with implants using a surgical guide fabricated using CT cross-sections and a computerized planning program.

\section{CASE REPORT}

A healthy 28-year-old female patient presented with an aggressive periodontal disease. Radiographic and clinical examination revealed that all her teeth were hopeless (Figs 1A and B) and the treatment plan consisted in extractions of all her teeth and at the end of the treatment, 


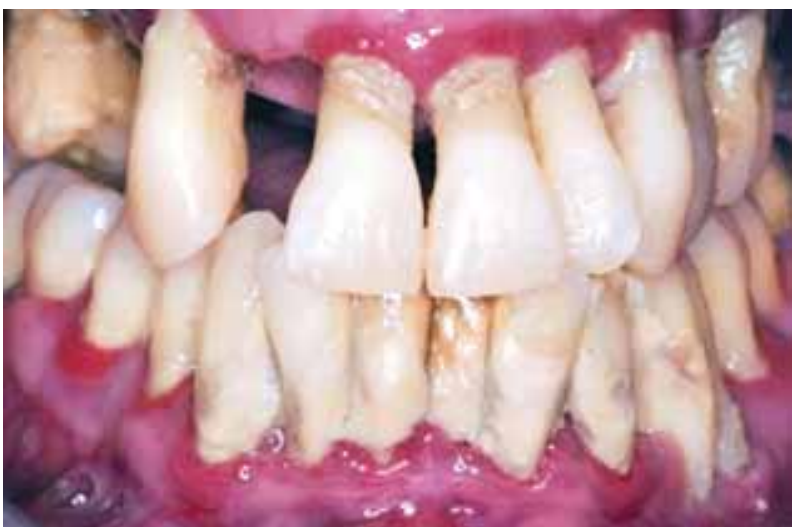

Fig. 1A: Preoperative clinical appearance showing advanced periodontal disease

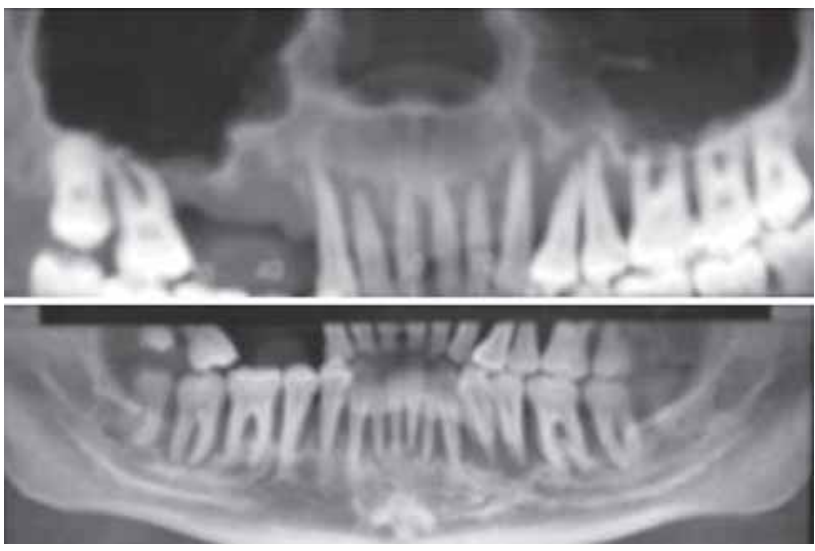

Fig. 1B: Radiographic appearance showing severe bone loss

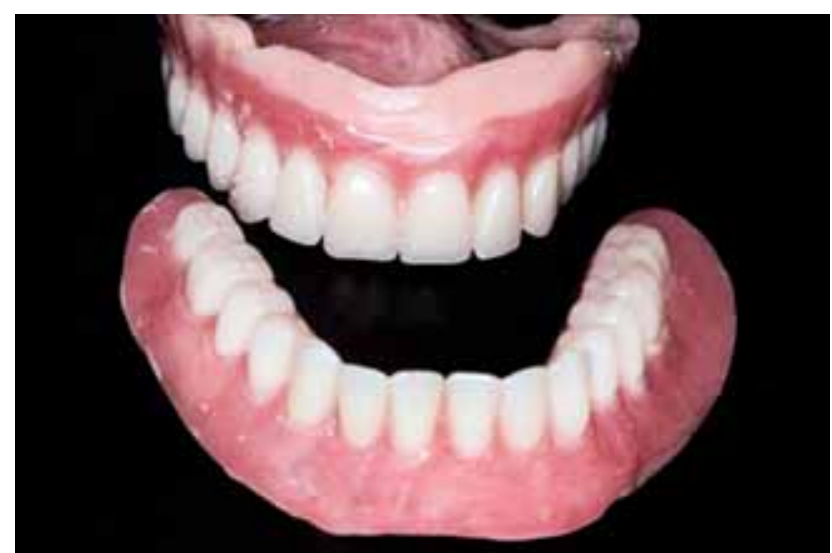

Fig. 2A: Upper and lower immediate complete dentures

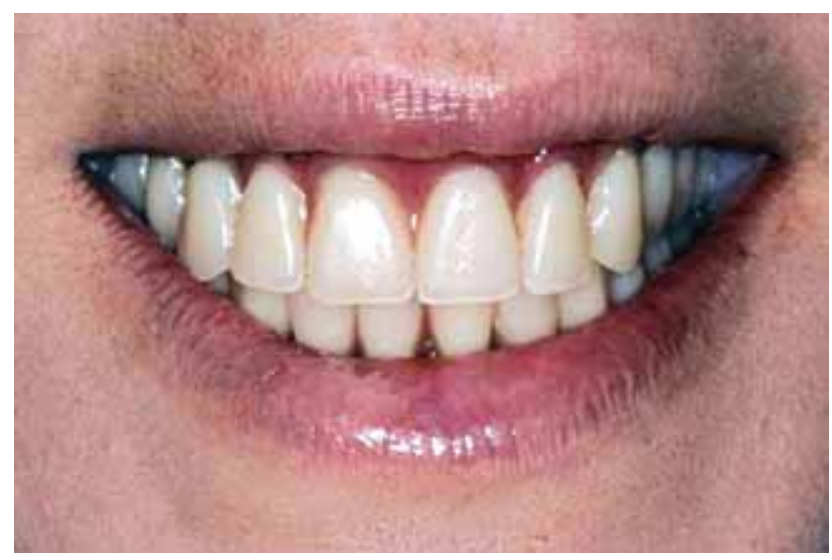

Fig. 2B: Clinical appearance of the dentures after delivery

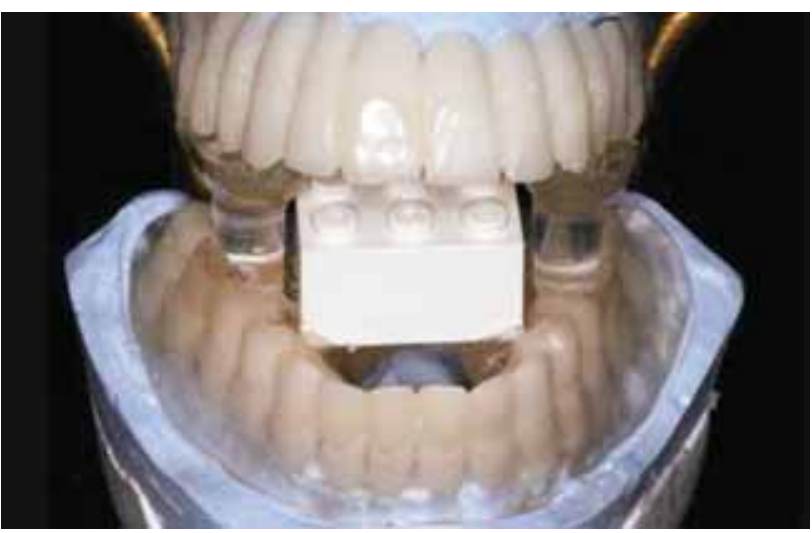

Fig. 3: Radiographic templates mounted on an articulator

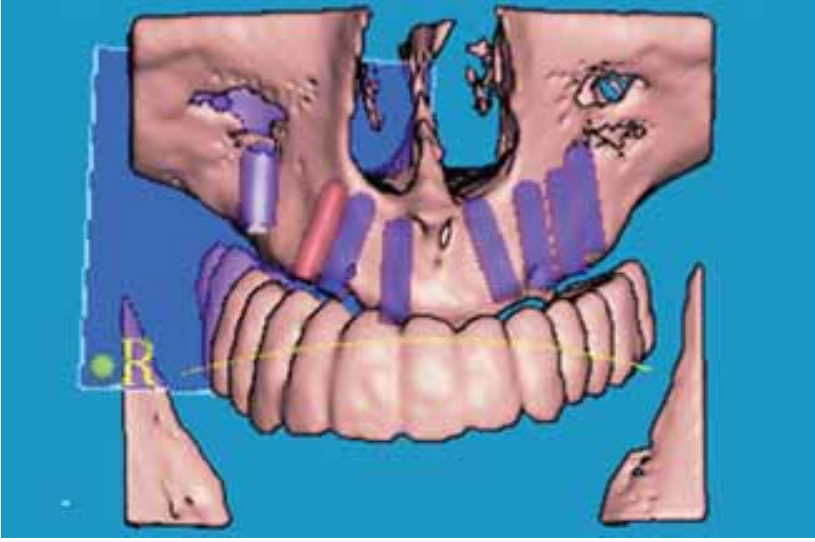

Fig. 4: Virtual planning of implants on the computerized reconstructed 3D model

delivery of maxillary and mandibular implant-supported fixed complete dentures. Immediately after the extractions, maxillary and mandibular complete removable dentures were delivered (Figs 2A and B). Following an esthetical and functional analysis, the dentures were duplicated and served as radiographic templates worn during the CT scan. During duplication, radiopaque barium sulfate powder was added to the diagnostic teeth. This allows for a precise evaluation of teeth positions on the resulting CT images. In addition, a registration cube was attached to the template (Fig. 3) for later 3D matching. The resulting CT images were exported to a viewing and implant planning software (Med3D, Heidelberg, Germany) and the clinician could immediately proceed to implant planning. After the available bone and other prosthodontic treatment criteria were evaluated, the locations and types of implants were selected. The implants were mounted virtually on the selected sections and the angulations, depths and diameters were assessed (Fig. 4).

Parallelism of the implants was also controlled and corrected. Once implant selection and positioning have been determined, the system converts the radiographic template to a surgical guide with incorporated drill housing (Fig. 5). After anesthesia, the mandibular surgical guide was secured over the untouched mandible by transfixing the guide flanges through gingival tissues to the bone using bone drills as stabilizers of the guide (Fig. 6). Six implants (Touareg, Adin 


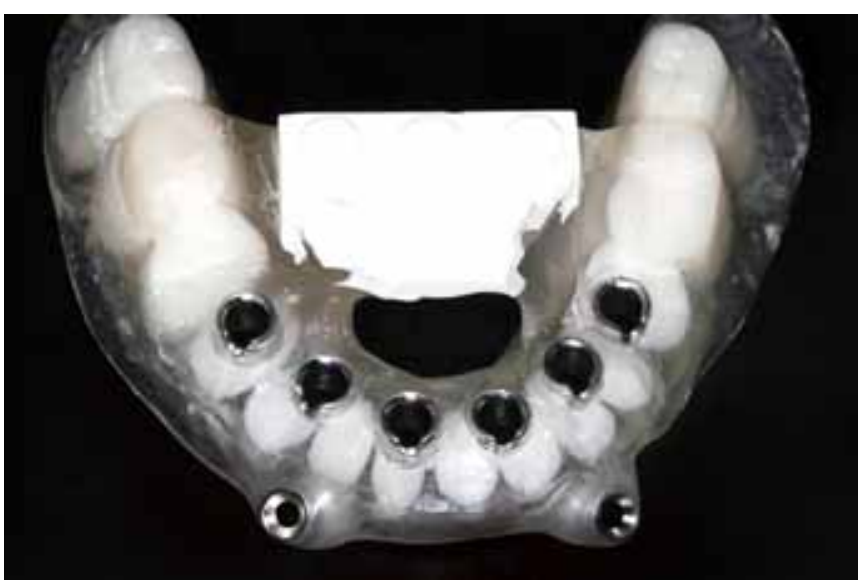

Fig. 5: Mandibular surgical guide with incorporated drill house

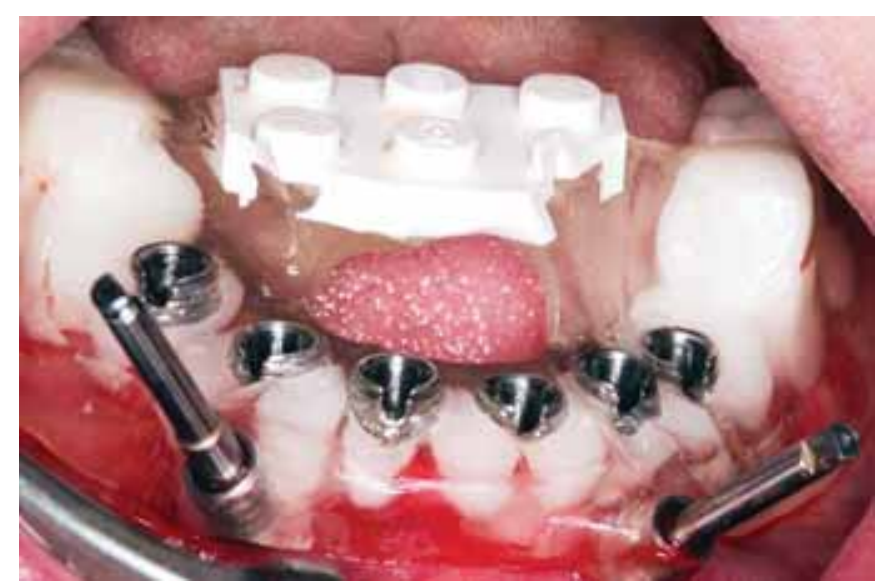

Fig. 6: Mandibular surgical guide stabilized by transfixing buccal screws

Alon-Tavor, Israel) were placed at the interforamina area with a flapless surgical approach, and were immediately loaded with a provisional restoration. The criteria for flapless surgery were: (1) Adequate amount of bone for implant placement, presence of $1 \mathrm{~mm}$ of bone buccolingual to the planned implant in a favorable prosthetic position as determined using the CT analysis, and (2) sufficient attached mucosa present at the surgical site such that at least $2 \mathrm{~mm}$ of attached gingiva would remain around the implant site circumferentially. A bilateral sinus lift procedure (Cerabone, Biomaterials GmbH, Dieburg, Germany) was performed and six months later seven implants (Touareg, Adin, Alon-Tavor, Israel) were placed with a flapless surgical approach using the same CAD/CAM surgical guidance system as used for the mandible (Fig. 7). Full-arch maxillary and mandibular impressions were made from polyether impression material (Genic, Sultan Healthcare, Hannover, Germany). The maxillary porcelain-fused-to-metal screw-retained final restoration was made with pink and white porcelain, taking in consideration, for the vertical dimension, the patient's high-lip-smile and, for the anterior-posterior horizontal dimension, the patient's need for an adequate lip-support (Figs $8 \mathrm{~A}$ and $\mathrm{B}$ ). The mandibular restoration was a porcelain-fused-to-metal, cement-retained fixed-denture (Fig. 9). The final prostheses were fitted and the occlusion

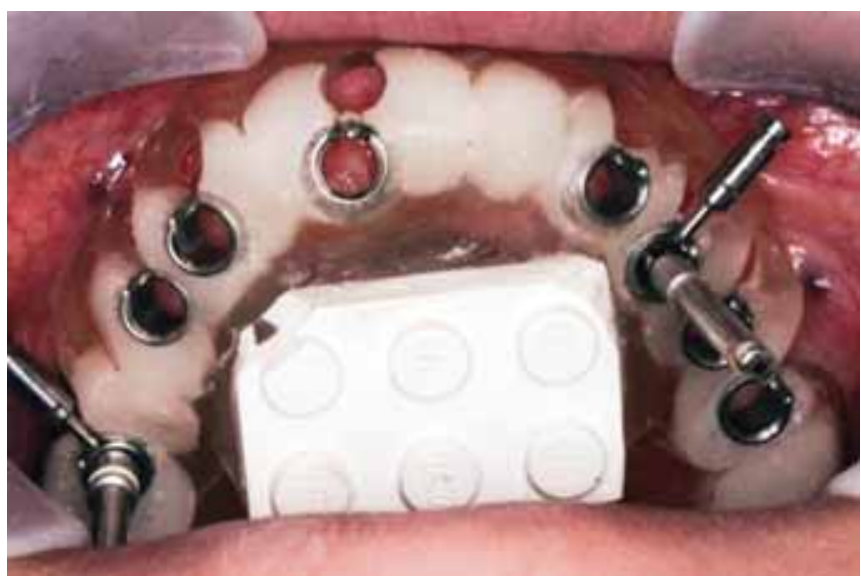

Fig. 7: Maxillary surgical guide in situ

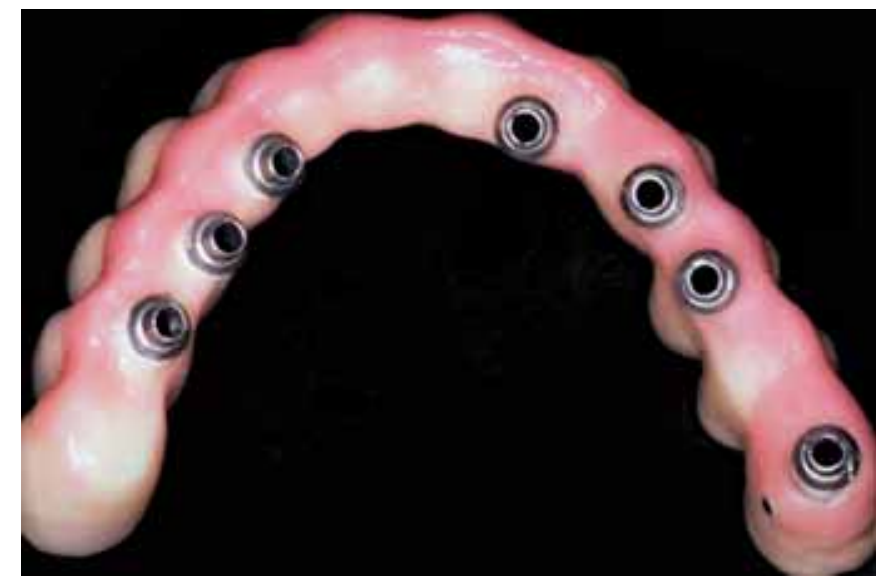

Fig. 8A: Maxillary PFM implant-supported-screwed restoration with cervical pink esthetics and buccal porcelain extension for lip support

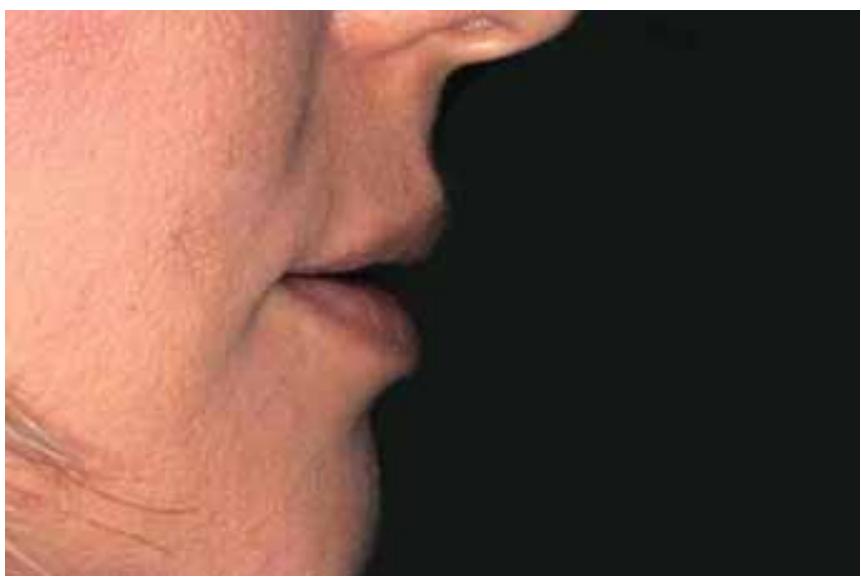

Fig. 8B: Patient's profile with the restoration in situ showing adequate lip support

was adjusted avoiding lateral contacts on cantilevers and passive-fit was verified radiographically (Figs 10A and B).

\section{DISCUSSION}

Flapless implant surgery is becoming accepted as an alternative protocol for placing dental implants.

Surgical templates fabricated using CT data allow the accurate transfer of a presurgical implant plan in flapless 


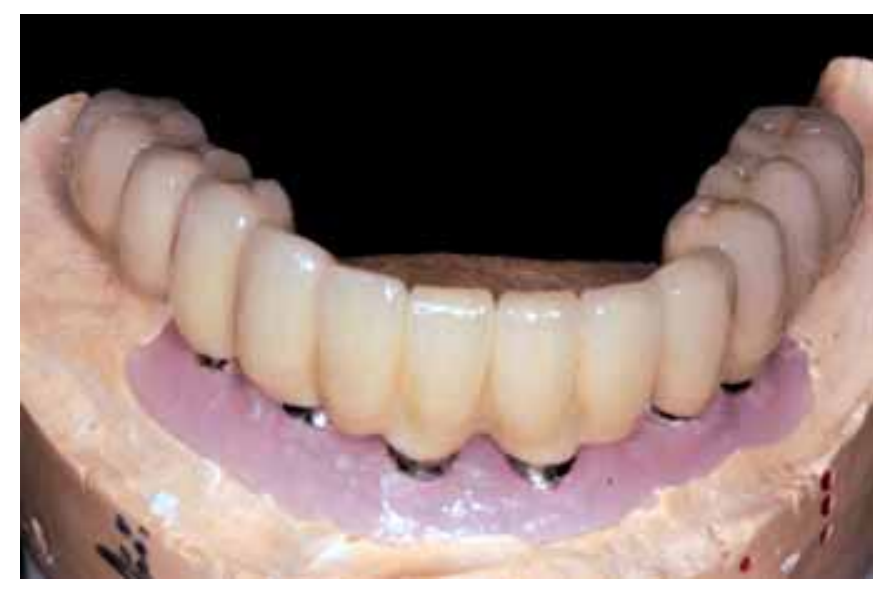

Fig. 9: Mandibular PFM implant-supported bonded restoration before delivery

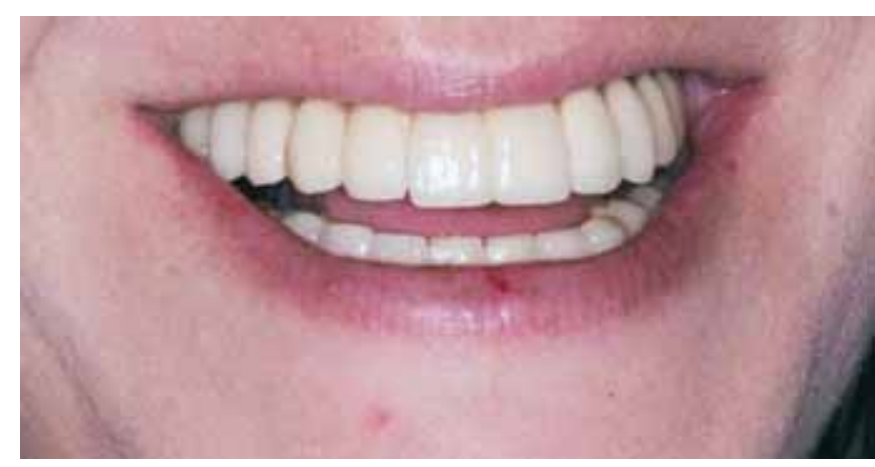

Fig. 10A: Clinical-frontal appearance of final seated restorations

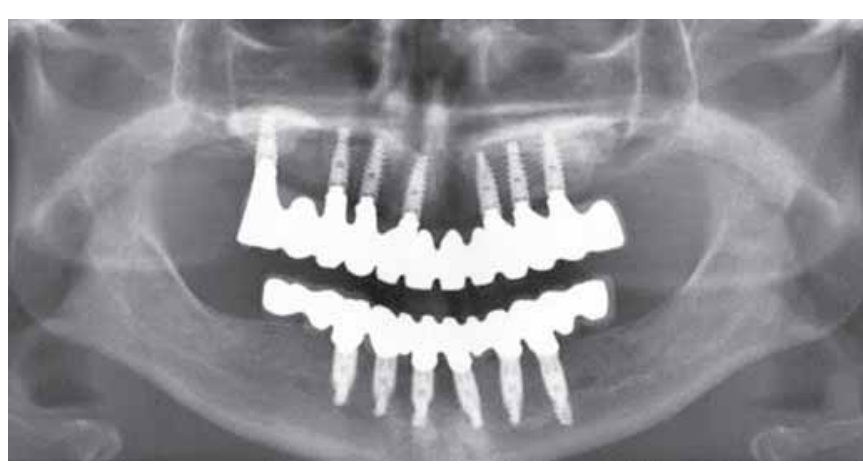

Fig. 10B: Postoperative panoramic X-ray verifying prostheses fit

implant surgery. The computer-based surgical guide can be used in completely as well as partially edentulous situations. The surgical guides can be supported by soft tissues, by bone, or by remaining teeth.

The benefits of the 3D CT software technique are apparent for the surgeon, the prosthodontist and the patient. ${ }^{11-14}$ It may also be possible to predict and quantify initial implant stability and bone quality from presurgical diagnosis using routine CT scan data and implant simulation software. ${ }^{15}$ The use of a radiographic guide during the CT scan optimizes scanned information and provides a reference during computer-aided implant placement. The use of a barium coating enables the buccolingual positioning of the implant to be correlated with the tooth outline.
Although there are many possible benefits to this technique, some limitations have to be taken in consideration. During the surgery, if the guide is not fixed horizontally to the bone with special pins, there is a risk of improper seating of the guide, which will result in misalignment of the implants, especially in the mandible. ${ }^{16}$ The guides also make it difficult to achieve and maintain the necessary coolness of the site during the surgery. It is also vital to evaluate the depth of each site during the surgery phase. The height of the mucosa, implants and the guiding steel ring must be accurately calculated, otherwise the cavity may be too shallow to accommodate the implant. Apart from these limitations, the technique is a very valuable tool in achieving successful results.

\section{CONCLUSION}

Extensive preoperative planning and treatment coordination are necessary for treatment success. Imaging tools, a diagnostic wax-up and a surgical guide, along with a good understanding of anatomy and surgical principles are essential. Early recognition of problem etiology and prompt treatment may prove to be invaluable to clinicians. Within the limitation of this complex case, clinical results seem to support the case that CT-based software and laboratorybased surgical guides may be used to decrease the incidence of implant-associated complications and to better assist the clinician in selecting and applying the most appropriate treatment options, such as flapless and implant's immediate loading. This technique also reduces chairside time and the healing time of the mucosa after surgery with minimal trauma to the oral tissues.

\section{REFERENCES}

1. Branemark PI, Svensson B, van Steenberghe D. Ten-year survival rates of fixed prostheses on four or six implants ad modum Branermark in full edentulism. Clin Oral Implants Res 1995;6:227-31.

2. Adell R, Eriksson B, Lekholm U, et al. Long-term follow-up study of osseointegrated implants in the treatment of totally edentulous jaws. Int J Oral Maxillofac Implants 1990;5: 347-59.

3. Jemt T, Lekholm U. Implant treatment in edentulous maxilla: A 5-year follow-up report on patients with different degrees of jaws resorption. Int J Oral Maxillofac Implants 1995;10: 303-11.

4. Campelo LD, Camara JR. Flapless implant surgery: A 10-year clinical retrospective analysis. Int J Oral Maxillofac Implants 2002;17:271-76.

5. Fortin T, Bosson JL, Isidori M, Blanchet E. Effect of flapless surgery on pain experienced in implant placement using an image guided system. Int J Oral Maxillofac Implants 2006;21:305-13.

6. Casap N, Tarazi E, Wexler A, Sonnenfield U, Lustman J. Intraoperative computerized navigation for flapless implant surgery and immediate loading in the edentulous mandible. Int J Oral Maxillofac Implants 2005;20:92-98. 
7. Jacobs R, Adriansens AS, Verstreken K, Suetens P, van Steenberghe D. Predictability of a three-dimensional planning system for oral implant surgery. Dentomaxillofac Radiol 1999;28:105-11.

8. Jabero M, Sarment DP. Advanced surgical guidance technology: A review. Implant Dent 2006;15:135-42.

9. Sarment D, Al-Shammari K, Kazor CE. Stereolithographic surgical templates for placement of dental implants in complex cases. Int J Periodontics Restorative Dent 2003;23:287-95.

10. Fortin T, Champleboux G, Bianchi S, Buatois H, Coudert JL. Precision of transfer of preoperative planning for oral implants based on cone-beam CT-scan images through a robotic drilling machine. Clin Oral Implants Res 2002;13:651-56.

11. Lal K, White SW, Morea DN, Wright RF. Use of stereolithographic templates for surgical and prostodontic implant planning and placement (Part 1). The concept. J Prosthodont 2006;15:51-58.
12. Parel SM, Triplett RG. Interactive imaging for implant planning, placement and prosthetic construction. J Oral Maxillofac Surg 2004;62:41-47.

13. Sarment DP, Sukovic P, Clinthorne N. Accuracy of implant placement with a stereolithograpic surgical guide. Int J Oral Maxillofac Implants 2003;18:571-77.

14. van Steenberghe D, Malevez C, van Cleynenbrengel J, et al. Accuracy of drilling guides for transfer from three-dimensional CT-based planning to placement of zygoma implants in human cadavers. Clin Oral Implant Res 2003;14:131-36.

15. Ikumi N, Tsutsumi S. Assesment of correlation between computerized tomography values of the bone and cutting torque values at implant placement: A clinical study. Int J Oral Maxillofac Implants 2005;20:253-60.

16. Di Giacomo GAP, Cury PR, de Araujo NY, Sendyk WR, Sendyk CL. Clinical application of stereolithographic surgical guides for implant placement: Preliminary results. J Periodontol 2005;76:503-07. 